\title{
Determining the divergence of an ultra-relativistic electron beam from the diffracted transition radiation in a single-crystal target
}

\author{
S.V. Blazhevich ${ }^{\mathrm{a}, *}$, M.V. Bronnikova ${ }^{\mathrm{a}}$, A.V. Noskov ${ }^{\mathrm{a}, \mathrm{b}}$ \\ a Belgorod State University, 85 Pobeda Str., Belgorod 308015, Russia \\ b Belgorod State Technological University named after V.G. Shukhov, 46 Kostyukov Str., Belgorod, 308012, Russia
}

\section{A R T I C L E I N F O}

\section{Article history:}

Received 17 October 2019

Received in revised form 20 January 2020

Accepted 6 February 2020

Available online 10 February 2020

Communicated by V.A. Markel

\section{Keywords:}

Relativistic electron

Single crystal

Angular density

Divergent beam of relativistic electrons

Diffracted transition radiation

Beam divergence determination

\begin{abstract}
A B S T R A C T
We have studied the possibilities of using diffracted transition radiation excited by a relativistic electron beam in a single-crystal target to determine the beam divergence. For this purpose, we investigated the expressions we had previously derived that describe the angular density of diffracted transition radiation excited by a relativistic electron beam crossing a thin single-crystal plate. In our calculations, we used the two-parameter model distribution function to represent the angular distribution of ultra-relativistic electrons in the beam. For determining the beam divergence parameters, the methods of two-dimensional objective function minimization were used. The model calculations we performed confirm the efficiency of the above-mentioned methods for determining the divergence of a relativistic electron beam.
\end{abstract}

(C) 2020 Elsevier B.V. All rights reserved.

\section{Introduction}

The problem of creating new methods for indicating beam parameters in charged particle accelerators is always relevant. The transverse size and divergence are base parameters of the accelerated electron beam. The main task of beam indication for the physicists creating, studying and applying the beams of relativistic electrons in the energy range of $100-1000 \mathrm{MeV}$ is the measurement of the beam transverse dimensions, as the divergence is $0.001 \mathrm{mrad}$ on up-to-date accelerators which is a negligible value for the beam transverse size exceeding $10 \mathrm{~mm}$. Currently, in the world there are two large electron-positron colliders [1,2] under construction. In these setups, the electrons and positrons will be accelerated up to $250 \mathrm{GeV}$ and will move in a beam of an extremely small size $(\sim 5-100 \mathrm{~nm})$. In this case, the main task of the beam indication will be the measurement of its divergence. The solution of this problem will allow a more informative and exact analysis of the experimental data in fundamental and applied investigations. It is evident that the measurements of electron beam divergence require methods minimally affecting the measured parameters. The use of electromagnetic radiation generated by relativistic electrons in a thin target is one of the promising directions in the development of the beam indication methods. Recently, the

\footnotetext{
* Corresponding author.

E-mail address: blazh@bsu.edu.ru (S.V. Blazhevich).
}

possibilities of using the parametric X-ray radiation (PXR) for diagnosing the transverse dimensions of the relativistic electron beam has been studied experimentally in $[3,4]$.

The effect of the divergence of an electron beam on PXR in a crystal was experimentally researched in Tomsk and Tokyo [5] for electron energies of 600 and $800 \mathrm{MeV}$, respectively. It was shown that the dependence of PXR on the orientation of the crystal is sensitive to the divergence of the beam, and it was proposed to use PXR as a simple tool for determining the angular divergence of beams of high-energy charged particles. In [6], it was proposed to use parametric X-ray radiation generated in thin crystals to obtain operational information about the position and size of an electron beam. In addition to PXR, in the direction of Bragg scattering, diffracted transition radiation (DTR) was also observed [7-10], which is a consequence of diffraction on a system of parallel atomic planes of a crystal of transition radiation photons generated on the front boundary of the crystal plate.

In our works [11-13] we developed the DTR theory for a monocrystalline target and for a periodic layered one considering the asymmetry of the Bragg reflection of the Coulomb field of a relativistic electron relative to the target surface. In [14], we developed a dynamic theory of coherent X-ray radiation by a divergent beam of relativistic electrons crossing a single-crystal plate in the Laue scattering geometry. The expressions obtained in [14] suggest that the angular density of the DTR depends on the divergence of the electron beam to a greater extent than the angular density of PXR. 
In the present work, the DTR generated by a beam of relativistic electrons in a single-crystal target in the Laue scattering geometry is studied in terms of its applicability for indicating the parameters of electron beams. We consider the radiation for a very thin target, when the multiple scattering of electrons on target atoms is negligible, which is important for measuring the divergence of an electron beam, as this case provides a sufficiently small change in the measured parameters. Since the contribution of PXR to the yield of coherent radiation in the conditions under consideration is negligible compared with the contribution of DTR, it is not considered in the present work.

In the paper, we analyze the possibility of using the DTR for indicating the divergence of relativistic electron beams of ultrahigh energies. We propose a method for defining the parameters of the two-dimensional angular distribution of electrons in the beam from the measured angular distribution of the diffracted transition radiation.

\section{Radiation process geometry}

In order to describe the radiation generated by a beam of relativistic electrons traversing a single-crystal plate we consider the radiation by one singled out electron in the beam. For the considered case of relativistic electrons, we are able to use the smallangle approximation in radiation process geometry. Each of the electrons in the beam will be determined by the position of its velocity vector $\mathbf{V}$ (Fig. 1) relative to the beam axis (unit vector $\mathbf{e}_{1}$ ) using the variable $\psi$ :

$\mathbf{V}=\left(1-\frac{1}{2} \gamma^{-2}-\frac{1}{2} \psi^{2}\right) \mathbf{e}_{1}+\psi, \quad \mathbf{e}_{1} \psi=0$,

where $\psi$ is the angle of the electron velocity deviation from the axis of the electron beam $\mathbf{e}_{1}$. This variable can be decomposed by components parallel and perpendicular to the plane of the picture in Fig. 1: $\psi=\psi_{\|}+\psi_{\perp}$.

The unit vector $\mathbf{n}$ of the momentum of the incident photon generated by this relativistic electron will be determined by its position relative to $\mathbf{e}_{1}$ using the angular variable $\boldsymbol{\theta}_{0}$ :

$\mathbf{n}=\left(1-\frac{1}{2} \theta_{0}^{2}\right) \mathbf{e}_{1}+\boldsymbol{\theta}_{0}, \quad \mathbf{e}_{1} \boldsymbol{\theta}_{0}=0$,

where $\boldsymbol{\theta}_{0}$ is the angle between the direction of the incident photon $\mathbf{n}$ and the axis $\mathbf{e}_{1}$.

Also, we will determine the unit vector $\mathbf{n}_{\mathbf{g}}$ of the diffracted photon momentum by its position relative to $\mathbf{e}_{2}$ using the vector angular variable $\boldsymbol{\theta}$ :

$\mathbf{n}_{\mathbf{g}}=\left(1-\frac{1}{2} \theta^{2}\right) \mathbf{e}_{2}+\boldsymbol{\theta}, \quad \mathbf{e}_{2} \boldsymbol{\theta}=0, \quad \mathbf{e}_{1} \mathbf{e}_{2}=\cos 2 \theta_{B}$.

The variable $\boldsymbol{\theta}$ is the angle of radiation, counted from the detector axis $\mathbf{e}_{2}$ (Bragg scattering direction). The angular variables determining the incident photon and the photon diffracted in the single-crystal target on the system of parallel atomic planes can be decomposed by components parallel and perpendicular to the plane of the picture (Fig. 1): $\boldsymbol{\theta}=\boldsymbol{\theta}_{\|}+\boldsymbol{\theta}_{\perp}, \boldsymbol{\theta}_{0}=\boldsymbol{\theta}_{0 \|}+\boldsymbol{\theta}_{0 \perp}$.

In Fig. $1, \psi_{0}$ is the electron beam divergence, $\theta_{B}$ is the Bragg scattering angle (defined under $\left.\boldsymbol{\theta}_{0}=0, \boldsymbol{\theta}=0\right), \gamma=1 / \sqrt{1-V^{2}}$ is Lorenz-factor of the electron, $\delta$ is the angle between the entrance boundary of the target and the system of diffracting atomic planes in the target. In formulas (1), we use the fact that the variables $\boldsymbol{\theta}_{0}$, $\boldsymbol{\theta}$, and $\psi$ are of the same order of smallness as $\gamma^{-1}\left(\gamma^{-1}<10^{-3}\right)$.

So, we will consider the coherent radiation of a separate electron in the beam crossing a single-crystal target at the angle

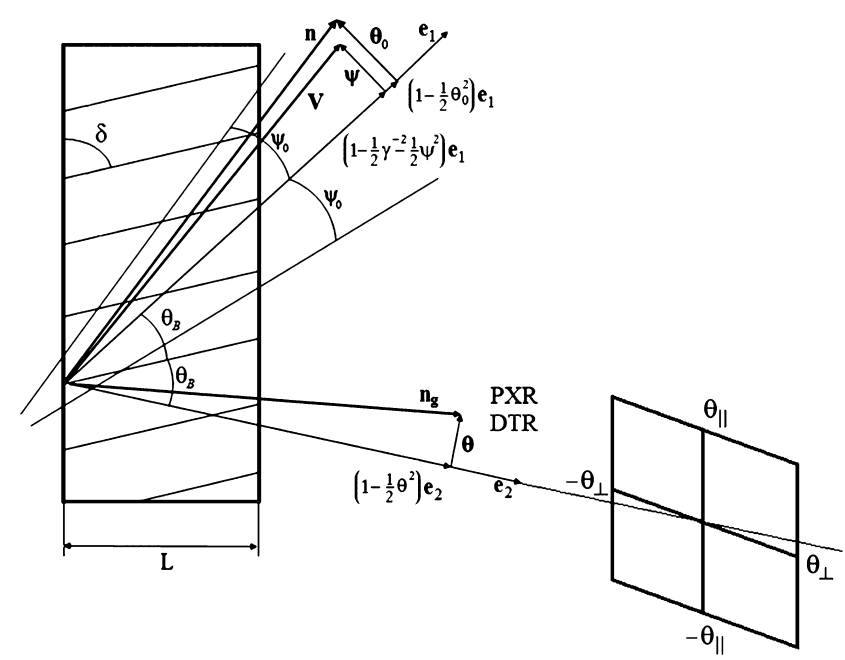

Fig. 1. The radiation process geometry.

$\boldsymbol{\psi}\left(\psi_{\|}, \psi_{\perp}\right)$ to the electron beam axis $\mathbf{e}_{1}$. To move from the radiation by a single electron to the one by a beam of electrons we will use the averaging of the radiation spectral-angular density by all the electron trajectories (over angles $\boldsymbol{\theta}=\boldsymbol{\theta}_{\|}+\boldsymbol{\theta}_{\perp}$ and $\left.\boldsymbol{\theta}_{0}=\boldsymbol{\theta}_{0 \|}+\boldsymbol{\theta}_{0 \perp}\right)$ as shown below.

The two main radiation mechanisms which contribute to the coherent radiation generated by a relativistic electron are the diffracted transition radiation (DTR) and the parametric X-ray radiation (PXR). Note that at super high energies of the electron which is under our consideration the contribution of PXR can be neglected.

\section{Spectral-angular density of DTR from a thin single-crystal plate}

We shall use the expression for spectral-angular density of DTR generated by an electron in the beam traversing a monocrystalline plate of arbitrary thickness obtained in [14]:

$$
\begin{aligned}
\omega \frac{d^{2} N_{D T R}^{(s)}}{d \omega d \Omega}= & \frac{e^{2}}{4 \pi^{2}} \Omega^{(s) 2} \times\left(\frac{1}{\gamma^{-2}+\left(\theta_{\perp}-\psi_{\perp}\right)^{2}+\left(\theta_{\|}+\psi_{\|}\right)^{2}}\right. \\
& \left.-\frac{1}{\gamma^{-2}+\left(\theta_{\perp}-\psi_{\perp}\right)^{2}+\left(\theta_{\|}+\psi_{\|}\right)^{2}-\chi_{0}^{\prime}}\right)^{2} R_{D T R}^{(s)}
\end{aligned}
$$

where the spectral function

$$
\begin{aligned}
R_{D T R}^{(s)}= & \frac{\varepsilon^{2}}{\xi(\omega)^{2}+\varepsilon} \\
& \times\left[\exp \left(-2 b^{(s)} \rho^{(s)} \Delta^{(1)}\right)+\exp \left(-2 b^{(s)} \rho^{(s)} \Delta^{(2)}\right)\right. \\
& \left.-2 \cdot \exp \left(-b^{(s)} \rho^{(s)} \frac{1+\varepsilon}{\varepsilon}\right) \cdot \cos \left(\frac{2 b^{(s)} \sqrt{\xi^{(s) 2}+\varepsilon}}{\varepsilon}\right)\right], \\
\Omega^{(1)}= & \theta_{\perp}-\psi_{\perp}, \quad \Omega^{(2)}=\theta_{\|}+\psi_{\|}, \\
C^{(1)}= & 1, \quad C^{(2)}=\cos 2 \theta_{B}, \\
\Delta^{(2)}= & \frac{\varepsilon+1}{2 \varepsilon}+\frac{1-\varepsilon}{2 \varepsilon} \frac{\xi^{(s)}}{\sqrt{\xi^{(s)^{2}}+\varepsilon}}+\frac{\kappa^{(s)}}{\sqrt{\xi^{(s)^{2}}+\varepsilon}} \\
\Delta^{(1)}= & \frac{\varepsilon+1}{2 \varepsilon}-\frac{1-\varepsilon}{2 \varepsilon} \frac{\xi^{(s)}}{\sqrt{\xi^{(s)^{2}}+\varepsilon}}-\frac{\kappa^{(s)}}{\sqrt{\xi^{(s)^{2}}+\varepsilon}},
\end{aligned}
$$




$$
\begin{aligned}
& \varepsilon=\frac{\sin \left(\delta+\theta_{B}\right)}{\sin \left(\delta-\theta_{B}\right)}, \quad \rho^{(s)}=\frac{\chi_{0}^{\prime \prime}}{\left|\chi_{\mathbf{g}}^{\prime}\right| C^{(s)}} \\
& b^{(s)}=\frac{1}{2 \sin \left(\delta-\theta_{B}\right)} \frac{L}{L_{e x t}^{(s)}}, \quad \nu^{(s)}=\frac{\chi_{\mathbf{g}}^{\prime} C^{(s)}}{\chi_{0}^{\prime}}, \quad \kappa^{(s)}=\frac{\chi_{\mathbf{g}}^{\prime \prime} C^{(s)}}{\chi_{0}^{\prime \prime}} \\
& \xi^{(s)}(\omega)=\eta^{(s)}(\omega)+\frac{1-\varepsilon}{2 \nu^{(s)}}, \\
& \eta^{(s)}(\omega)=\frac{2 \sin ^{2} \theta_{B}}{V^{2}\left|\chi_{\mathbf{g}}^{\prime}\right| C^{(s)}}\left(\frac{\omega\left(1-\theta_{\left./ / \cot \theta_{B}\right)}\right.}{\omega_{B}}-1\right) \\
& d \Omega \equiv d \theta_{\|} \cdot d \theta_{\perp} .
\end{aligned}
$$

The expressions (2) describe $\sigma$-polarized waves under $s=1$ and $\pi$-polarized waves under $s=2$. The expressions (2) describe the spectral-angular density of DTR taking into account the deviation of the electron velocity direction (the angle $\psi\left(\psi_{\perp}, \psi_{/ /}\right)$) in relation to the electron beam axis $\mathbf{e}_{1}$. These expressions have been derived within the frame of a two-wave approximation [15] of the dynamical diffraction theory for the general case of the asymmetric reflection of the radiation waves in relation to the front surface of the monocrystalline target (angle $\delta$ ). Parameter $\varepsilon$ determines the degree of asymmetry of the reflection of the coulomb field of the relativistic electron relative to the target surface. It should be noted that the angle of the electron incidence to the target surface $\left(\delta-\theta_{B}\right)$ decreases when asymmetry parameter $\varepsilon$ increases. Parameter $b^{(s)}$ is equal to half the path of the electron in target $L_{e}=L / \sin \left(\delta-\theta_{B}\right)$ expressed in the lengths of extinction of X-ray waves in crystal $L_{\text {ext }}^{(s)}=1 / \omega\left|\chi_{\mathbf{g}}^{\prime}\right| C^{(s)}$.

Now we shall consider the radiation excited by the relativistic electrons in a thin nonabsorbing crystal, i.e. under the condition when the longest path of the diffracted photon in plate $L_{f}=L / \sin \left(\delta+\theta_{B}\right)$ is considerably shorter than the length of $\mathrm{x}$ ray absorption in the crystal $L_{a b s}=1 / \omega \chi_{0}^{\prime \prime}$ :

$2 \frac{b^{(s)} \rho^{(s)}}{\varepsilon}=\frac{L_{f}}{L_{a b s}} \ll 1$.

For the case of a thin nonabsorbing target the spectral function (2b) takes the form

$R_{D T R}^{(s)}=\frac{4 \varepsilon^{2}}{\xi^{(s) 2}+\varepsilon} \sin ^{2}\left(b^{(s)} \frac{\sqrt{\xi^{(s) 2}+\varepsilon}}{\varepsilon}\right)$.

To determine the angular density of DTR we should integrate the expression (2a) over $\omega$ using frequency function $\xi^{(s)}(\omega)$ and the relationship $\frac{d \omega}{\omega}=\frac{\left|\chi_{\mathbf{g}}^{\prime}\right| C^{(s)}}{2 \sin ^{2} \theta_{B}} d \xi^{(s)}$ from the definition of $\xi^{(s)}(\omega)$ in (3). Then we will obtain the DTR angular density in the following form:

$$
\begin{aligned}
\frac{d N_{D T R}^{(s)}}{d \Omega}= & \frac{e^{2} \chi_{0}^{\prime 2}}{8 \pi^{2} \sin ^{2} \theta_{B}\left|\chi_{\mathbf{g}}^{\prime}\right| C^{(s)}} \times \frac{\Omega^{(s) 2}}{\sigma^{(s) 2}\left(\left|\chi_{\mathbf{g}}^{\prime}\right| C^{(s)} \sigma^{(s)}+\chi_{0}^{\prime}\right)^{2}} \\
& \times \int_{-\infty}^{\infty} R_{D T R}^{(s)} d \xi^{(s)}(\omega),
\end{aligned}
$$

where

$$
\sigma^{(s)}=\frac{1}{\left|\chi_{g}^{\prime}\right| C^{(s)}}\left(\gamma^{-2}+\left(\theta_{\perp}-\psi_{\perp}\right)^{2}+\left(\theta_{/ /}+\psi_{/ /}\right)^{2}-\chi_{0}^{\prime}\right) \text {. }
$$

Further we consider the case $b^{(s)} \ll \sqrt{\varepsilon}$. The spectral function in (6) integrated over frequency $\omega$ by using approximation (5) leads to the following expression for integral:

$$
\int_{-\infty}^{\infty} R_{D T R}^{(s)} d \xi^{(s)}(\omega) \approx 4 \pi \varepsilon b^{(s)}, \quad b^{(s)} \ll \sqrt{\varepsilon} .
$$

In this case the expression for the angular density of the DTR takes the form

$$
\begin{aligned}
\frac{d N_{D T R}^{(s)}}{d \Omega}= & \frac{e^{2} \omega_{B} \chi_{0}^{\prime 2} \chi_{\mathbf{g}}^{\prime 2} C^{(s) 2}}{4 \pi \sin ^{2} \theta_{B}} \frac{\Omega^{(s) 2}}{\left(\gamma^{-2}+\Omega^{2}-\chi_{0}^{\prime}\right)^{2} \cdot\left(\gamma^{-2}+\Omega^{2}\right)^{2}} \\
& \times \varepsilon \frac{L}{\sin \left(\delta-\theta_{B}\right)},
\end{aligned}
$$

where $\Omega^{2}=\left(\theta_{\perp}-\psi_{\perp}\right)^{2}+\left(\theta_{/ /}+\psi_{/ /}\right)^{2}$.

Now we can use the obtained formula (8) for determining the ultrarelativistic electron beam divergence parameters from the measured distribution of the angular density of the DTR generated in a thin $\left(b^{(s)} \ll \sqrt{\varepsilon}\right)$ single-crystal target. This expression is derived within the framework of the two-wave approximation of the dynamic theory of diffraction taking into account the deviation of the electron velocity direction (angle $\boldsymbol{\psi}$ ) from beam axis $\mathbf{e}_{1}$ (see in Fig. 1).

\section{The angular density of the DTR excited by a divergent beam of relativistic electrons}

Let us consider the influence of the divergence of the beam of super high energy electrons traversing a thin monocrystalline target on the DTR angular density. For this purpose, we average the expression for the DTR angular density over all possible rectilinear trajectories of the electron in the beam. As an example, we carry out the averaging of the DTR angular density (8) over the two-dimensional Gauss distribution

$f(\psi)=\frac{1}{\pi \psi_{0 \perp} \psi_{0 \|}} e^{-\left(\frac{\psi_{\perp}^{2}}{\psi_{0 \perp}^{2}}+\frac{\psi_{\|}^{2}}{\psi_{0 \|}^{2}}\right)}$,

where $\psi_{0 \|}$ and $\psi_{0 \perp}$ are the parameters of the electrons beam divergence in the plane of the mirror reflection of the coulomb field of the electron on a system of parallel atomic planes in the crystalline target and in the plane perpendicular to it (see in Fig. 1) accordingly. Parameters $\psi_{0 \|}$ and $\psi_{0 \perp}$ define an elliptical cone bounding the part of the electron beam beyond which the angular density of the electron distribution decreases by more than $e$ times compared with the density on the beam axis. In the considered case the expression for the averaged DTR angular density generated by the beam of relativistic electrons normalized by the number of electrons, takes the form

$$
\begin{aligned}
& \left\langle\frac{d N_{D T R}^{(s)}}{d \Omega}\right\rangle_{b^{(s)} \ll \sqrt{\varepsilon}}=\frac{e^{2} \omega_{B} \chi_{\mathbf{g}}^{\prime 2}}{4 \pi \sin ^{2} \theta_{B}} \frac{\varepsilon L}{\sin \left(\delta-\theta_{B}\right)} \frac{1}{\pi \psi_{0 \perp} \psi_{0 \|}} \\
& \quad \times \int_{-\infty}^{\infty} \int_{-\infty}^{\infty} \frac{\Omega^{(s) 2} e^{-\left(\frac{\psi_{\perp}^{2}}{\psi_{0 \perp}^{2}}+\frac{\psi_{\|}^{2}}{\psi_{0 \|}^{2}}\right)}}{\left(\gamma^{-2}+\Omega^{2}-\chi_{0}^{\prime}\right)^{2} \cdot\left(\gamma^{-2}+\Omega^{2}\right)^{2}} d \psi_{\perp} d \psi_{\|} .
\end{aligned}
$$

In Fig. 2 the curves plotted by formula (10) demonstrate the distribution of the angular density of the DTR generated by the electron beam of a different divergence. In Fig. 3 the curves are plotted for analogous conditions except the energy of electrons which are considerably higher than in the case of Fig. 2.

The curves shown in Fig. 2 and Fig. 3 demonstrate the significant dependence of the DTR angular density on the divergence of the electron beam. Fig. 2 illustrates that the angular density of the DTR under the considered parameters of the monocrystalline target for the electron energy $E_{e}=5 \mathrm{GeV}$ is substantially sensitive to the beam divergence in the range $\psi_{0} \leq 0.2 \mathrm{mrad}$.

Under the energy of beam electron $E_{e}=100 \mathrm{GeV}$ (see Fig. 3) the DTR angular density is sensitive to electron beam divergence in the range $\psi_{0} \leq 0.02 \mathrm{mrad}$. One can see that under higher electron 


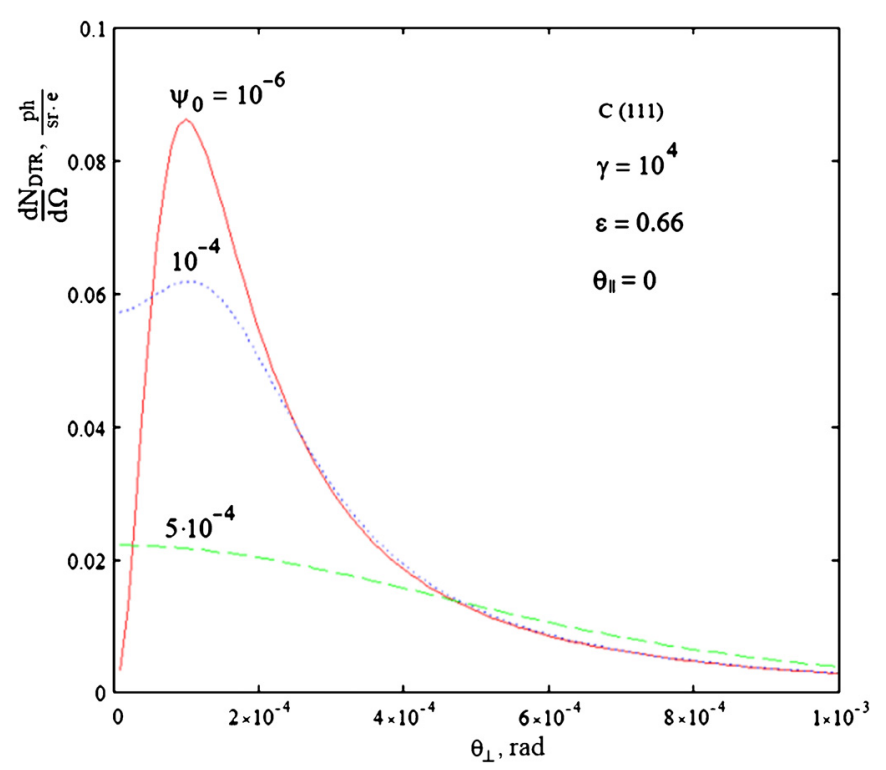

Fig. 2. The dependence of the DTR angular density on the divergence of the relativistic electron beam generating it. The distribution of the DTR angular density on angle $\boldsymbol{\theta}_{\perp}$ in the plane perpendicular to the plane of Fig. 1 containing axis $\mathbf{e}_{2}$ (see in Fig. 1) for different values of the beam divergence $\psi_{0}=\sqrt{\psi_{0 \perp}^{2}+\psi_{0 \|}^{2}}$ is demonstrated. The electron energy $E_{e}=5 \mathrm{GeV}\left(\gamma=10^{4}\right)$, the angle $\boldsymbol{\theta}_{\|}=0$.

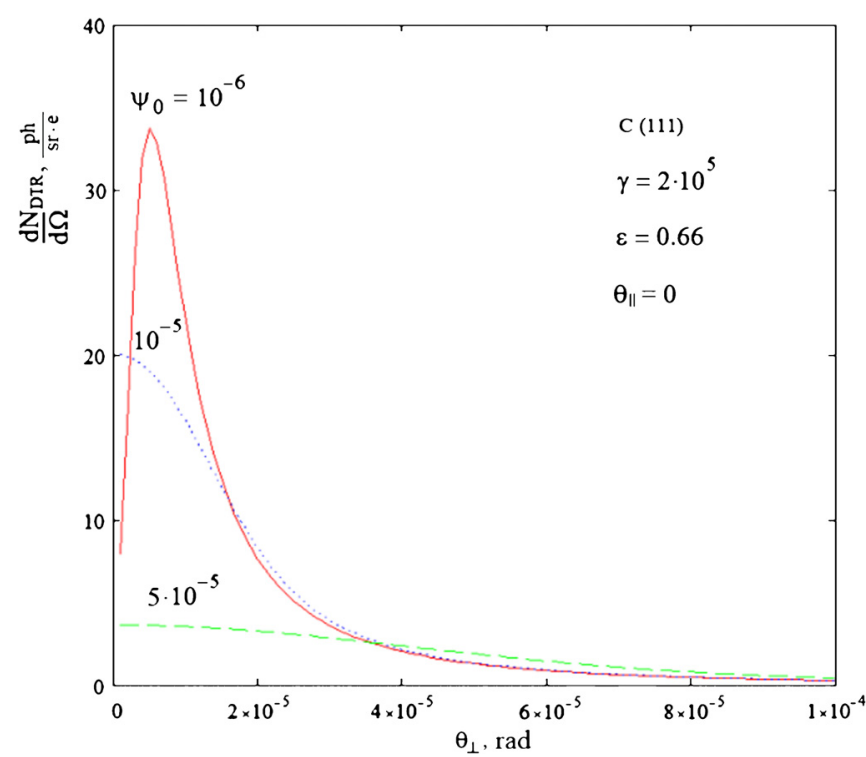

Fig. 3. The same as in Fig. 2 but for the value of electron energy about $E_{e}=100$ $\mathrm{GeV}\left(\gamma=2 \cdot 10^{5}\right)$.

energy the DTR angular distribution becomes narrower, and the angular density becomes more sensitive to the divergence of the electron beam.

\section{Determination of the electron beam divergence parameters using the measured angular distribution of the DTR}

The divergence of a relativistic electron beam can be estimated based on the DTR angular density by using the approximation of the DTR angular distribution by a function with one or several parameters. For example, for the approximation of the angular distribution of electrons in the beam we shall use the bivariate normal distribution (9) with the values of distribution dispersions $\psi_{0 \|}$ and $\psi_{0 \perp}$ (beam divergence parameters) which are different in horizontal and vertical planes.
In this work variants of using the angular distribution of the DTR generated by an electron beam of super high energy in a thin nonabsorbing monocrystalline target for indicating the beam divergence parameters shall be demonstrated. As an example of a single-crystal target we shall give consideration to a thin plate of diamond with diffracting atomic planes (111).

In order to determine the divergence parameters, we shall solve the task of objective function minimization. As the objective function, the mean-square deviation of the calculated DTR angular distribution from the measured one in the experiment is used.

We shall solve this problem by fitting the parameters of the normal angular distribution in one or two orthogonal planes containing the electron beam axis, in particular, in the plane of the mirror reflection of the coulomb field of the relativistic electron from a system of atomic planes (111) of the diamond single-crystal target.

For detecting the opportunities of the minimization process optimization, such as, reducing the calculation time and minimizing the error of determining the divergence parameters, we have considered two variants for resolving this problem.

In the first variant, we use as the objective function the sum of the mean-square deviations of the calculated and measured DTR angular density for a row of $N$ points of angular density distribution $\left(\theta_{\perp i}, \theta_{/ /}=0\right)$, containing the axis $\mathbf{e}_{2}\left(\theta_{\perp}=0, \theta_{/ /}=0\right)$, where $i=1,2 . . N$ (see in Fig. 1 ):

$$
\begin{aligned}
\varphi 0\left(\psi_{0 \perp}, \psi_{0 / /}\right)= & \sum_{i}^{N}\left(\frac{d N_{D T R}}{d \Omega}\left(\theta_{\perp i}, \theta_{/ / i}, \psi_{0 \perp}, \psi_{0 / /}\right)\right. \\
& \left.-\frac{d N_{D T R}}{d \Omega}\left(\theta_{\perp i}, \theta_{/ / i}\right)^{E X P}\right)^{2},
\end{aligned}
$$

where

$$
\frac{d N_{D T R}}{d \Omega}\left(\theta_{\perp i}, \theta_{/ / i}, \psi_{0 \perp}, \psi_{0 / /}\right)=\sum_{s=1}^{2}\left(\frac{d N_{D T R}^{(s)}}{d \Omega}\left(\theta_{\perp i}, \theta_{/ / i}, \psi_{0 \perp}, \psi_{0 / /}\right)\right),
$$

$(s)$ is the index of polarization vector projection on the coordinate axes, $N$ - the number of points presenting DTR angular distribution in the chosen interval of $\theta_{\perp}$. The value of $\theta_{/ /}$is chosen as $\theta_{/ /}=0$. As "measured" values of angular density, the values of the DTR angular density calculated by (10) for the given values of the parameters of the electron beam divergence $\psi_{0 \perp}=1 / \gamma$ and $\psi_{0 / /}=2 / \gamma$ are used. In reality, the "points" of the measured distribution of the DTR angular density will be of a finite size. At the angular distribution interval of $-2 / \gamma \leq \theta_{\perp}<2 / \gamma$ under $\theta_{\|}=0$ the dependence of the objective function on the parameters of divergence has a pronounced minimum, that defines the unambiguity in searching the optimal parameters value (see in Fig. 4).

We solved the task of the two-dimensional objective function minimization in two different ways - the method of Hook-Jeeves [16] and the Nelder-Mead method [17].

The Hook-Jeeves minimization method proved to be preferable for this purpose as it cuts the computation 1.5 times relative to the Nelder-Mead method and allows better control of the calculation intermediate result errors.

In the second variant of the solution of the divergence parameters determination problem we use the objective function constructed as the sum of square deviations of the measured and calculated values of the DTR angular density in three characteristic points of the angular density distribution $\left(\theta_{\perp}=0, \theta_{\|}=0\right)$, $\left(\theta_{\perp}=\gamma^{-1}, \theta_{\|}=0\right)$ and $\left(\theta_{\perp}=0, \theta_{\|}=\gamma^{-1}\right)$. These points correspond to the minimal and maximal values of the DTR angular density generated by one electron moving in the target along the electron beam axis. 


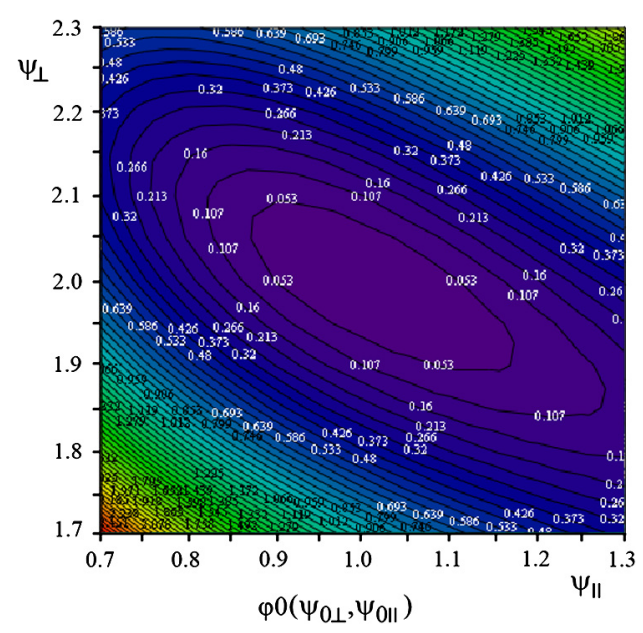

Fig. 4. Two-dimensional objective function (11), whose minimal value corresponds to the desired divergence parameters of the relativistic electron beam $\psi_{0 \perp}=$ $\left(\psi_{0 \perp}\right)_{\exp }$ and $\psi_{0 \|}=\left(\psi_{0 \|}\right)_{\exp }$.

So, we search for the parameters of the beam divergence $\psi_{0 \perp}$ and $\psi_{0 \|}$ by minimizing the objective function:

$$
\varphi\left(\psi_{0 \perp}, \psi_{0 / /}\right)=\varphi 1\left(\psi_{0 \perp}, \psi_{0 / /}\right)+\varphi 2\left(\psi_{0 \perp}, \psi_{0 / /}\right)
$$$$
+\varphi 3\left(\psi_{0 \perp}, \psi_{0 / /}\right) \text {, }
$$
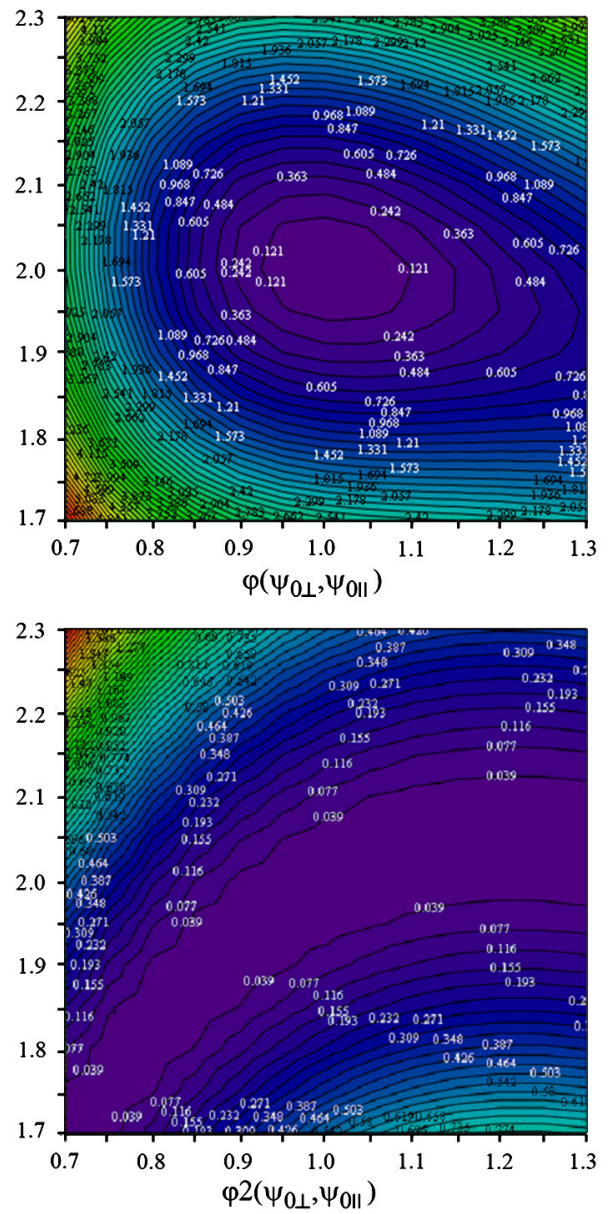

where

$$
\begin{aligned}
& \varphi 1\left(\psi_{0 \perp}, \psi_{0 / /}\right)=\left(\frac{d N_{D T R}}{d \Omega}\left(\theta_{\perp}=0, \theta_{/ /}=0, \psi_{0 \perp}, \psi_{0 / /}\right)\right. \\
&\left.-\frac{d N_{D T R}}{d \Omega}\left(\theta_{\perp}=0, \theta_{/ /}=0\right)^{E X P}\right)^{2}, \\
& \varphi 2\left(\psi_{0 \perp}, \psi_{0 / /}\right)=\left(\frac{d N_{D T R}}{d \Omega}\left(\theta_{\perp}=0, \theta_{/ /}=1 / \gamma, \psi_{0 \perp}, \psi_{0 / /}\right)\right. \\
&\left.-\frac{d N_{D T R}}{d \Omega}\left(\theta_{\perp}=0, \theta_{/ /}=1 / \gamma\right)^{E X P}\right)^{2}, \\
& \varphi 3\left(\psi_{0 \perp}, \psi_{0 / /}\right)=\left(\frac{d N_{D T R}}{d \Omega}\left(\theta_{\perp}=1 / \gamma, \theta_{/ /}=0, \psi_{0 \perp}, \psi_{0 / /}\right)\right. \\
&\left.-\frac{d N_{D T R}}{d \Omega}\left(\theta_{\perp}=1 / \gamma, \theta_{/ /}=0\right)^{E X P}\right)^{2}, \\
& \frac{d N_{D T R}}{d \Omega}\left(\theta_{\perp}, \theta_{\|}, \psi_{0 \perp}, \psi_{0 \|}\right)=\sum_{s=1}^{2}\left(\frac{d N_{D T R}^{(s)}}{d \Omega}\left(\theta_{\perp}, \theta_{\|}, \psi_{0 \perp}, \psi_{0\|\|}\right)\right) .
\end{aligned}
$$

This variant was chosen to reduce the calculation time. Toward approximating the angular distribution of an electron beam by two-dimension normal distribution we shall seek the optimal values of the parameters of distribution with computer programs implementing the minimization method of Hook-Jeeves. In pictures Fig. 5 the objective function and the contributions of each of
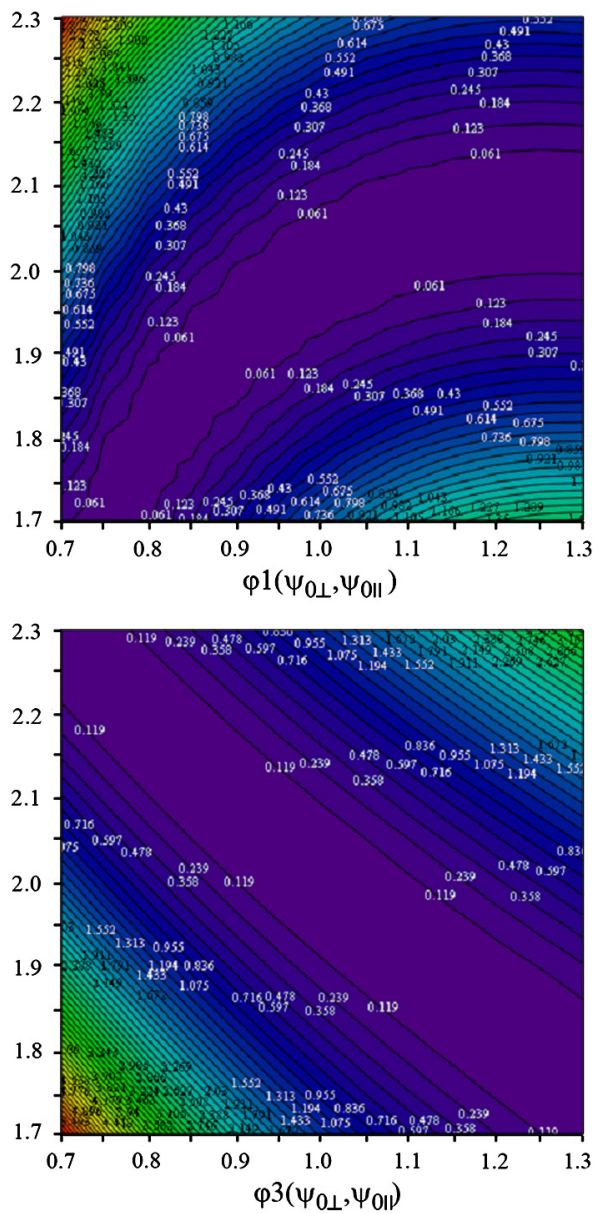

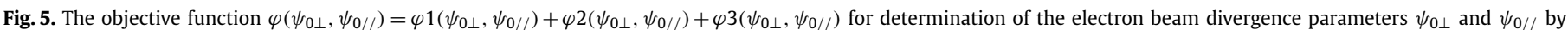

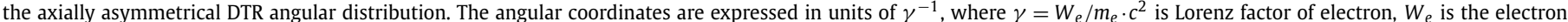

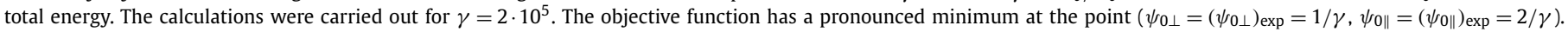

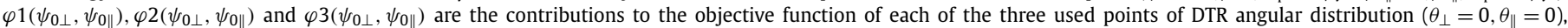
$\left(\theta_{\perp}=0, \theta_{\|}=\gamma^{-1}\right)$ and $\left(\theta_{\perp}=\gamma^{-1}, \theta_{\|}=0\right)$ correspondently. 
these three points of DTR distribution in the objective function are demonstrated. One can see that each of these contributions have ravine topology and cannot allow the exact defining of the minimum on the objective function, but their sum has a pronounced minimum, which provides the unequivocal determination of divergence parameters of a two-dimension angular distribution of the electron beam.

For example, the minimization process of the objective function was built on three points of the DTR angular distribution using the Hook-Jeeves method. For the "experimentally measured" value of angular density in each of the chosen points of the distribution the values calculated for the beam with divergence parameters $\psi_{0 \perp}=1 / \gamma$ and $\psi_{0 \|}=2 / \gamma$ are used. The approximation of the calculated parameter to these "experimental" values was carried out for the DTR generated by the beam of electrons $\left(\gamma=2 \cdot 10^{5}\right)$ on a system of atomic planes (111) of a thin $(L=50 \mu \mathrm{m})$ diamond single-crystal target.

The second variant provides the conditions for calculating the beam divergence parameters using the extremal small number of the points in the DTR angular density distribution that considerably saves the computation time.

It should be noted that in a real measuring system the measure "points" on the angular distribution of the DTR will be represented by dots of finite size, providing a set of statistical data enough to achieve the required measurement accuracy. Since the sensitivity of the DTR angular distribution to the changes in the electron beam parameters at these points is maximal, we expect the measurement error not to be greater than in the first method. The size of each of the "points" on the detector can be comparable with the characteristic radius $L_{D} \cdot \gamma^{-1}$, where $L_{D}$ is the distance from the target to the registration plane. This is possible because, while solving the task, we determine the parameters of a two-parametric distribution function, which is not intended to describe more detailed features of the real distribution of the electron beam density.

Another important parameter of the relativistic electron beam on the accelerator is its transverse size. This parameter is meant to measure exactly in the required place of the beam indication. In our case, we assume that the transverse size of the electron beam on the target is small compared to the size that it would have at a distance equal to the distance to the detector of diffracted transition radiation (DTR) and therefore insignificantly influences the accuracy of the beam divergence measurement.

\section{Conclusions}

The analytical expressions for the angular density of diffracted transition radiation (DTR) excited by a divergent beam of relativistic electrons in a thin monocrystalline target have been examined. The calculations of the angular density of the DTR emitted from a thin plate of diamond $C(111)$ as the target have been made. The modeling of the process of determining the divergence parameters for the relativistic electron beam from the measured distribution of the DTR angular density was implemented using the optimization method based on objective function minimiza- tion. The objective function was built in the form of a sum of squared deviations of the calculated values of the DTR angular density from the measured ones in the chosen points of the DTR angular distribution. We estimated the efficiency of the applied algorithms by calculating the beam divergence parameters using different two-dimensional objective functions and different methods of their minimization.

\section{Declaration of competing interest}

The authors declare that they have no known competing financial interests or personal relationships that could have appeared to influence the work reported in this paper.

\section{Acknowledgements}

The present work is supported by the Ministry of Education and Science of the Russian Federation (project of state task No 3.4877.2017/BY), Russia.

\section{References}

[1] ILC Technical Design Report, 12 June 2013.

[2] M. Aicheler, et al. (Eds.), A Multi-TeV Linear Collider Based on CLIC Technology, CLIC Conceptual Design Report, CERN, 2012, 841 p.

[3] Y. Takabayashi, Parametric X-ray radiation as a beam size monitor, Phys. Lett A 376 (2012) 2408

[4] Y. Takabayashi, K. Sumitani, New method for measuring beam profiles using a parametric X-ray pinhole camera, Phys. Lett. A 377 (2013) 2577.

[5] B.N. Kalinin, A.P. Potylitsin, V.A. Verzilov, I.E. Vnukov, et al., Possible application of PXR to the beam-divergence measurements, Nucl. Instrum. Methods Phys. Res., Sect. A 350 (1994) 601.

[6] A. Gogolev, A. Potylitsyn, G. Kube, A possibility of transverse beam size diagnostics using parametric X-ray radiation, J. Phys. Conf. Ser. 357 (2012) 012018.

[7] A. Caticha, Transition-diffracted radiation and the Cherenkov emission of $\mathrm{x}$ rays, Phys. Rev. A 40 (1989) 4322.

[8] V. Baryshevsky, Parametric X-ray radiation at a small angle near the velocity direction of the relativistic particle, Nucl. Instrum. Methods A 122 (1997) 13

[9] X. Artru, P. Rullhusen, Parametric X-rays and diffracted transition radiation in perfect and mosaic crystals, Nucl. Instrum. Methods B 145 (1998) 1.

[10] N. Nasonov, Influence of the density effect upon the parametric X-rays of high energy particles, Phys. Lett. A 246 (1998) 148

[11] S.V. Blazhevich, Yu.A. Boltenko, T.V. Koskova, A.A. Mazilov, A.V. Noskov, Influence of relativistic electron beam divergence on angular characteristics of PXR and DTR generated in a single-crystal plate in Bragg scattering geometry, Probl. At. Sci. Tech., Ser. Phys. Radiat. Eff. Radiat. Mat. Sci. 5 (99) (2015) 3-7.

[12] S.V. Blazhevich, A.V. Noskov, Coherent X-radiation of relativistic electron in a single crystal under asymmetric reflection conditions, Nucl. Instrum. Methods B 266 (2008) 3770

[13] S. Blazhevich, A. Noskov, Dynamic theory of coherent X-radiation of relativistic electron within a periodic layered medium in Bragg scattering geometry, Nucl. Instrum. Methods B 309 (2013) 70-75.

[14] S.V. Blazhevich, A.V. Noskov, Diffracted transition radiation of an ultra-highenergy relativistic electron beam in a thin single-crystal wafer, J. Exp. Theor. Phys. 123 (4) (2016) 551-556.

[15] B. Batterman, H. Cole, Rev. Mod. Phys. 36 (3) (1964) 681-717.

[16] R. Hooke, T.A. Jeeves, Direct search solution of numerical and statistical problems, J. ACM 8 (1961) 212-229.

[17] J.A. Nelder, R. Mead, A simplex-method for function minimization, Comput. J. 7 (1965) 308-313. 